\title{
EDITORS' NOTE: VOLUME OVERVIEW
}

\section{ABSTRACT}

This issue of the International Journal of Telerehabilitation (IJT) contains original research evaluating the efficacy of telerehabilitation; a randomized study measuring costs and outcomes of tele-intervention when serving families of children who are deaf/hard-of-hearing; and a telephone-based physiotherapy intervention for patients with osteoarthritis of the knee. A feasibility study on the use of e-supervision to support speech-language pathologist graduate students during student teaching practica outlines benefits, limitations, and implications for future practice. In addition to innovative research, this issue includes announcements from the American Telemedicine Association and the Mid-Atlantic Telehealth Resource Center.

\section{VOLUME OVERVIEW}

The current issue of the International Journal of Telerehabilitation (IJT) contains original research that investigates the efficacy and cost-effectiveness of using tele-intervention to deliver high quality early intervention services to families of children who are deaf/hard-ofhearing. In their randomized study, Blaiser and colleagues compared remote service delivery (tele-intervention) with in-person service delivery on outcomes associated with expressive language and parent engagement. Tele-intervention yielded statistically significant positive outcomes when compared to in-person services on the SKI-HI Language Development Scale (expressive language measure) and Home Visit Rating Scales-Adapted \& Extended (parent engagement measure). In addition to these outcomes, provider and caregiver perceptions of tele-intervention were reported as generally positive, and a cost analysis demonstrated substantial cost savings with the use of tele-intervention over a 24-month period.

Odole and Ojo investigated the use of telerehabilitation by physiotherapists in Nigeria to improve pain and physical functioning of patients with knee osteoarthritis. In their randomized study, the researchers compared pain ratings and physical functioning of 50 patients randomly assigned to a tele-physiotherapy group or clinic group. The tele-physiotherapy group received thrice weekly telephone-based interventions to support implementation of a self-administered exercise protocol; the clinic group received thrice weekly clinic-based interventions with the exercise protocol implemented by a physiotherapist. Comparable outcomes between the groups demonstrated that the use of telephonic methods may be a practical and effective means to manage knee osteoarthritis, particularly in parts of the world where videoconferencing technologies may not be available or widely used.
In their mixed method feasibility study, Carlin and colleagues demonstrated that SLP graduate students engaged in a student teaching practica received adequate supervision, feedback, and support through the use of e-supervision. Benefits, limitations, and implications for future practice are discussed.

\section{CALL FOR SUBMISSIONS}

The next volume of the International Journal of Telerehabilitation will be published in spring, 2014. We cordially invite your submissions by April 20, 2014 and accept original research, case studies, viewpoints, technology reviews, book reviews, and country reports that detail the current status of telerehabilitation.

Our peer reviewers constitute a multi-disciplinary group, and include researchers and clinicians from each of the major rehabilitation disciplines, rehabilitation engineers, health information managers, information technologists, and others. We welcome new peer-reviewers and invite guest editors with ideas for special, thematically focused issues. The IJT publication team is agile and can add issues as warranted to ensure currency. Please contact Editor Ellen Cohn, PhD (ecohn@pitt.edu) or Associate Editor Jana Cason (jcason@spalding.edu) if you are interested. 


\section{ACKNOWLEDGMENTS}

We acknowledge the contributions of our authors and the support of new and returning reviewers; colleagues at the Rehabilitation Research Engineering Center on Telerehabilitation; and our publishers, Timothy S.

Deliyannides, Director, Office of Scholarly Communication and Publishing and Head, Information Technology,

University Library System, and Vanessa Gabler, Electronic Publications Associate at the University of Pittsburgh.

Respectfully,

Jana Cason, DHS, OTR/L, FAOTA

Issue Co-Editor and IJT Associate Editor

Ellen R. Cohn, PhD, CCC-SLP

IJT Editor 